\title{
Flexible Design of EGB Structure for Antenna Appliations
}

\author{
Huynh Nguyen Bao Phuong'), Dao Ngoc Chien ${ }^{1)}$, and Tran Minh Tuan ${ }^{2)}$ \\ ${ }^{1)}$ School of Electronics and Telecommunications, Hanoi University of Science and Technology \\ ${ }^{2)}$ Ministry of Information and Communications of Socialist Republic of Vietnam \\ E-mail: phuonghnb-set@mail.hut.edu.vn
}

Abstract: In this paper, we present a flexible design of electromagnetic bandgap (EBG) structure, which is constructed based on Fractal geometry, for antenna applications. These Fractals, which are the Sierpinski triangles, are arranged to repeat each $60^{\circ}$ to introduce the hexagonal unit cells. By changing the gap between two adjacent Sierpinski triangles inside EBG unit cell, it can be introducing two EBG structuresseparately that have broadband and dual bandgap. By using the suspending microstrip method, two arrays $3 \times 4$ of EBG unit cells areutilized to investigate the bandgap of the EBG structures. The EBG operation bandwidth of the broadband structure and the dual-band structure are about $87 \%$ and $40 \% ; 35 \%$ at the center bandgap frequencies, respectively. Moreover, a comparison between the broadband EBG and the conventional mushroom-like EBG has been done. Experimental results of the proposed design show good agreement in comparison with simulation results. Finally, the proposed EBG structures are studied as a high impedance ground plane for enhancing the radiation properties of a patch antenna.

\section{INTRODUCTION}

In recent years, there has been growing interest in investigating meta-materials, such as high impedance surface (HIS) composed of metal or dielectric unit cells, can be observed due to their unique characteristics in antennas and microwave circuits applications[1-4]. One of the most important properties of EBG structure is to prohibit the propagation of the surface wave [5], [6]. Practical applications of EBG structure usually have difficulty in accommodating its physical size, because the period of EBG lattices has to be a half-wavelength at the bandgap frequency. This problem had not been solved until the mushroom-like EBG was proposed by D. Sievenpiper et al [7]. Then, several of novel EBG structures were presented such as spiral EBG, uniplanar compact EBG (UC-EBG), fork-like EBG. These structures have several advantages, such as compact size, low loss, and so on. However, due to their resonant property, all of those structures are narrow band, which makes them not practical for use in broadband applications. It is a real challenging task to create an EBG with wide bandwidth.

Several broadband EBG structures were found in the literature. Typically, there have been two approaches aiming to obtain EBG structures with wider bandwidth: to use EBGs with via-holes [8] (with the inconveniences of complex and expensive manufacturing process) and the adoption of multilayered FSSs over a metallic ground plane or multi-period mushroom-like structure [9] (which yields less compact designs and is rather expensive). Recent research efforts focus on the development of planar EBG structures that do not need via and that can be integrated antenna to enhance the gain and reduce the backward radiation and increasing efficiency [10-12].
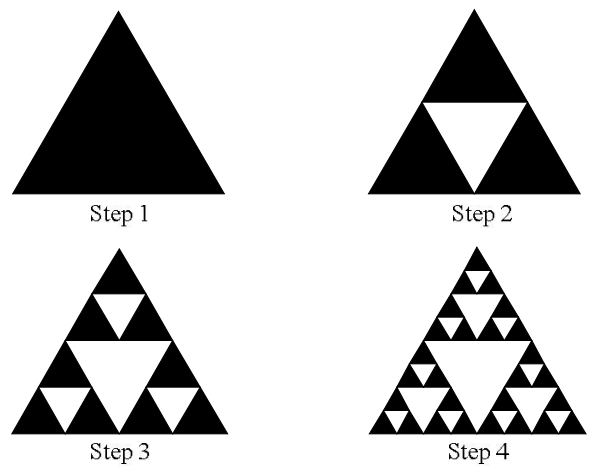

Figure 1. The four steps to introduce a mode-2 Sierpinski Gasket structure. 
As [13], an incomplete list of Fractal shapes can be used for antennas includes: 1) the von Koch curve; 2) the Minkowski curve; 3) the Hilbert curve; 4) the Fractal tree; 5) the Sierpinski (gasket and carpet) Fractal; and 6) the Cantor set. These geometries also can be used in order to create EBG structures. However, only the geometries those were creating multi-resonant frequencies are able to introduce broader bandgap. Actually, when an EBG structure has a multi-band, the broadband can be done by overlapping the different single-band for each other. In literature, Sierpinski (gasket and carpet) structures are widely used to create multi-band antenna [13]. From the idea of this design that is produced a hexagonal EBG with Fractal geometry, due to the characteristic of an equilateral triangle, Sierpinski gasket is chosen as a metal patchonthe top surface in order to create the hexagonal EBG. In this paper, the design of EBG structures involving the mode-2 Sierpinski Gasket triangles (see Fig. 1), and without via-holes or multilayer substrate is presented. The proposed EBG structures were simulated and measured using the method of suspended microstrip, which is recommended in [14] and their results were validated and found to be well correlated.

The rest of this paper is organized as follows. In Section II, the detailed designs of the novel EBG structures are presented. By using the suspending microstrip method, the scatter parameters of the proposed EBG structure based on Sierpinski triangle that is formed by different steps, are characterized and simulated in Section III. Besides, the bandgap of the conventional EBG structure is also determined for verifying the broader bandwidth of the proposed EBG structure. Next, the measurement of the proposed EBG structures in fabrication and discussions are also utilized in section IV. The application of the proposed EBG structures to a patch antenna is presented in section $\mathrm{V}$, while the conclusion is provided in Section VI.

\section{FRACTAL EBG STRUCTURE}

When the periodicity is small compared to the operating wavelength, the EBG structure can be simply as a parallel LC equivalent circuit. The resonant frequency of the EBG structure $f_{C}$ is calculated as follows:

$$
f_{C}=\frac{1}{2 \pi \sqrt{L C}}
$$

The bandwidth BW of the bandgap can be determined by the surface capacitance $C$ and inductance $L$ :

$$
B \mathrm{~W}=\frac{1}{\eta} \sqrt{\frac{L}{C}}
$$

Where $\eta$ is the free space impedance, which is $120 \pi$.

The designed unit cell geometry exhibits six symmetry planes, which makes it polarization-angle independent. The proposed EBG structures are shown in the Fig. 2, which are printed on a FR4 dielectric slab with dielectric constant of 4.4 and a thickness of $1.6 \mathrm{~mm}$ and loss tangent of 0.02. Dark parts in this figure represent the metallic periodic structure, which is etched on a dielectric substrate. Unit cell dimension is $W=10 \mathrm{~mm}$ and the metallization thickness is 18 $\mu \mathrm{m}$.

Several EBG structures based on Fractal geometry was presented in the literature [15], [16]. A multiband EBG, which is using Sierpinski Gasket was proposed in [15]. In this design, the EBG was formed by combining four Sierpinski triangles at step 1 and step 2. However, two of four triangular patches are connected to the solid lower ground plane by a metal plated via, which is leading to more complexity in the fabrication. Similarly, in [16] an EBG structure was designed by combining a metal patch with the Mandelbrot Fractal at the $2^{\text {nd }}$ iteration. As results, this EBG structure can produce multi-bandgap, but the limitation of this structure also uses metal via. Therefore, the planar EBG designing based on Fractal geometry is more comfortable to integrate with the antennas of the advanced wireless systems.

The Sierpinski gasket triangle in this paper is formed by the following process (see Fig. 2). Firstly, create an equilateral triangle patch with length edge of $W 1$. The second step of this Fractal EBG is constructed by etching out the center equilateral triangle of length edge $W 2$ inside the patch and 
Research, Development and Application on Information and Communication Technology

subtracting it from the patch. The next stage is achieved by subtracting additional three equilateral triangles of length edge $W 3$ of the center triangles that follows the same technique adopted to realize the mode-2 Sierpinski gasket geometry (see Fig. 2c).

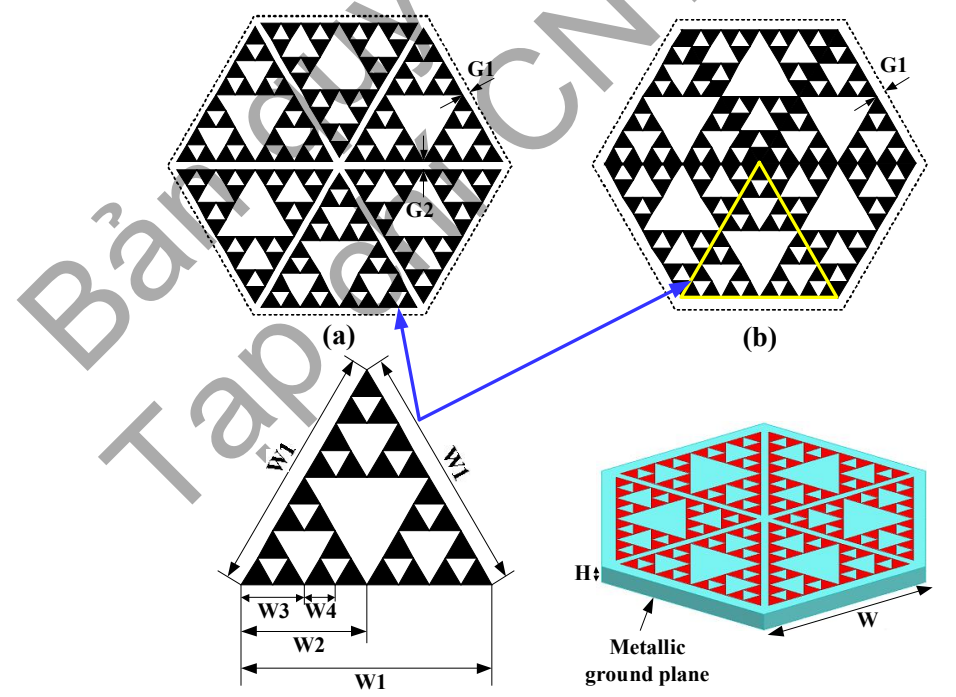

(c)

(d)

Figure 2. Unit cell geometries: (a) BEBG; (b) DEBG; (c) Detailed Sierpinski Gasket triangles; (d) Slide view of BEBG. Detailed dimensions: $W 4=W 1 / 8$; $W 3=W 1 / 4 ; W 2=W 1 / 2$;

$W 1=W-2(G, G 2=0.5 \mathrm{~mm}, G 1=1 \mathrm{~mm}$.

From this design, the Sierpinski gasket triangles are arranged to repeat each $60^{\circ}$. The aim of this work allows modifying the resonance frequencies and the EBG operation bandwidth by changing the width and the gap $G l$ between the hexagonal lattices. Moreover, by changing the gap G2 between Sierpinskitriangleunits, it can introduce two EBG structures separately. The first one, which is called broadband EBG structure (BEBG), has a broader bandgap with the value of $G 2$ up to $0 \mathrm{~mm}$. The second one, which is called dual-band EBG structure (DEBG), has dual bandgap when the value of $G 2$ is set at $0 \mathrm{~mm}$. These structures are shown in Fig. $2 \mathrm{a}$ and Fig.2b, respectively.

\section{THE BANDGAP CHARACTERISTICS}

In this section, the bandgap characteristics of two hexagonal EBG structures, which are formed by the Sierpinski triangle at $G_{2}=0$ and $G_{2}=0.5 \mathrm{~mm}$, are investigated. Besides, the bandgap of the conventional mushroom-like EBG is also determined for the comparison with the proposed EBG structures. In order to analyze the bandgap properties of these EBG structures with finite unit number, an experiment concerning transmission through the above structures has been carried out. A $3 \times 4$ conventional EBG structure and proposed EBG structures have been simulated using the method of suspended microstrip, which is proposed by Fanto measure the bandgap characteristic of the EBGs, as shown in Fig. 3 [11]. The operating frequency is set at $7 \mathrm{GHz}$, and the $50 \Omega$ microstrip line is placed on a dielectric support layer with the thickness of $0.5 \mathrm{~mm}$. The bandgap bandwidth will be defined with $\mathrm{S} 11$ is above $-5 \mathrm{~dB}$ and $\mathrm{S} 21$ is below $-30 \mathrm{~dB}$ at the same time.

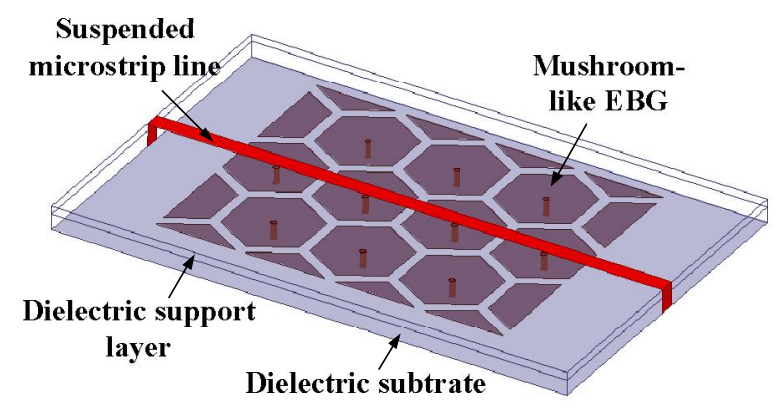

(a)

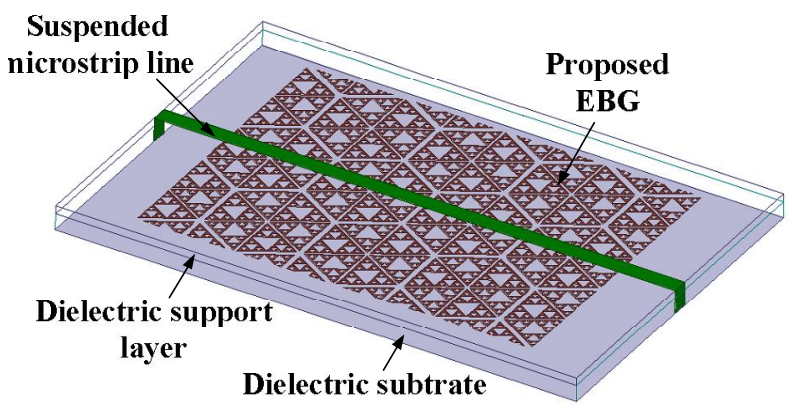

(b)

Figure 3. Geometry of $3 \times 4$ EBG arrays: (a) Mushroom-like EBG array, (b) Proposed EBG array. 


\section{A. EBG Structures at Different Steps}

The hexagonal EBG structures with Sierpinski triangles at different steps are investigated in the case of the value of $W$ is fixed at $10 \mathrm{~mm}$ while the value of $G 2$ is set at $0 \mathrm{~mm}$ and $0.5 \mathrm{~mm}$. The simulation results of the EBG structures based on the Sierpinski triangle at step 1, step 2, and step 3 are shown in Fig. 4, Fig. 5, and Fig. 6, respectively.

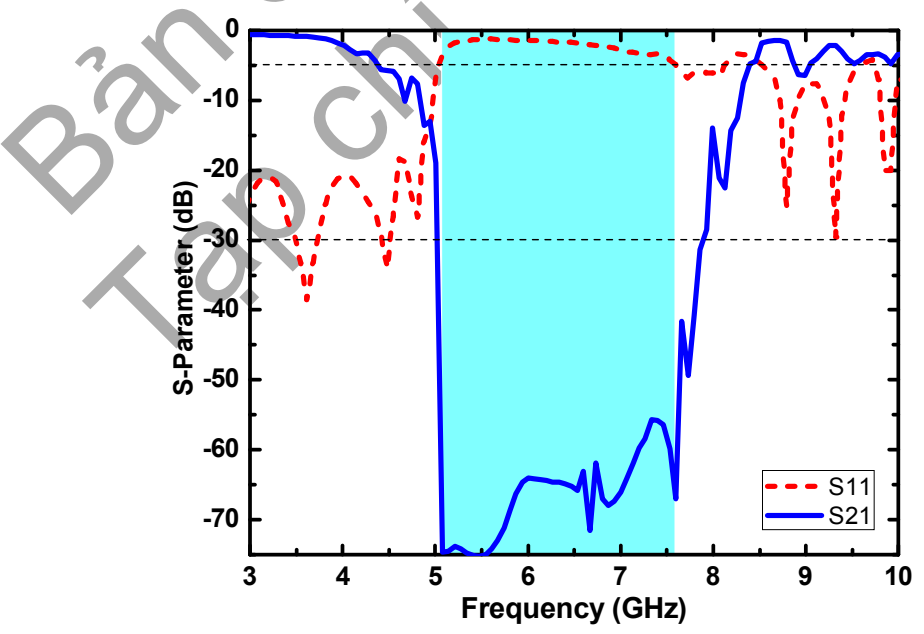

(a)

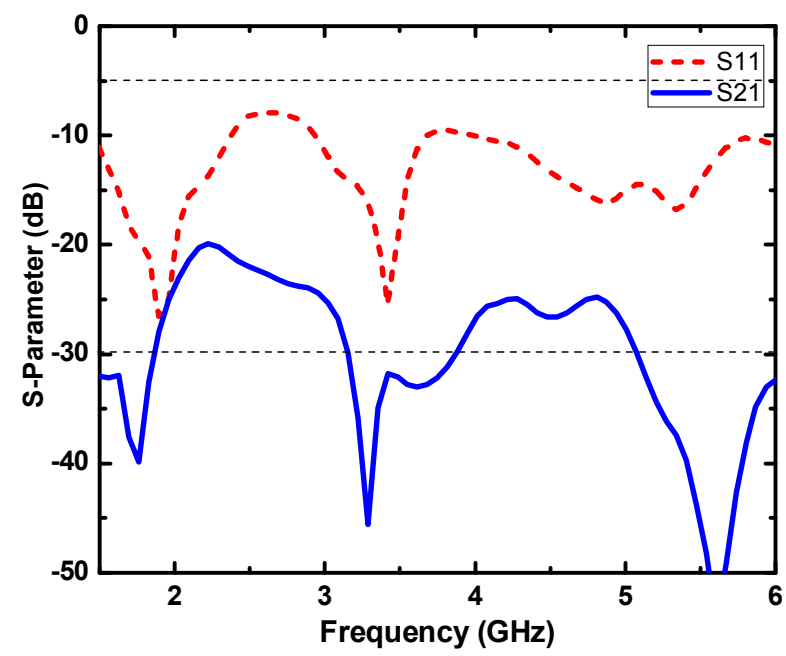

(b)

Figure 4. Operation bandwidth of Hexagonal EBG structure based onSierpinski triangle at step 1: a) G2 $=0.5 \mathrm{~mm}$ and b) $G 2=0 \mathrm{~mm}$
Step 1: As can be seen in Fig. 4a, the EBG structure introduces a bandgap, which is ranging from $5.07 \mathrm{GHz}$ to $7.58 \mathrm{GHz}$, as the $G 2$ is equal to $0.5 \mathrm{~mm}$. However, the bandgapwas not found in the case of $G 2$ value is equal to $0 \mathrm{~mm}$ (see Fig. $4 \mathrm{~b}$ ).

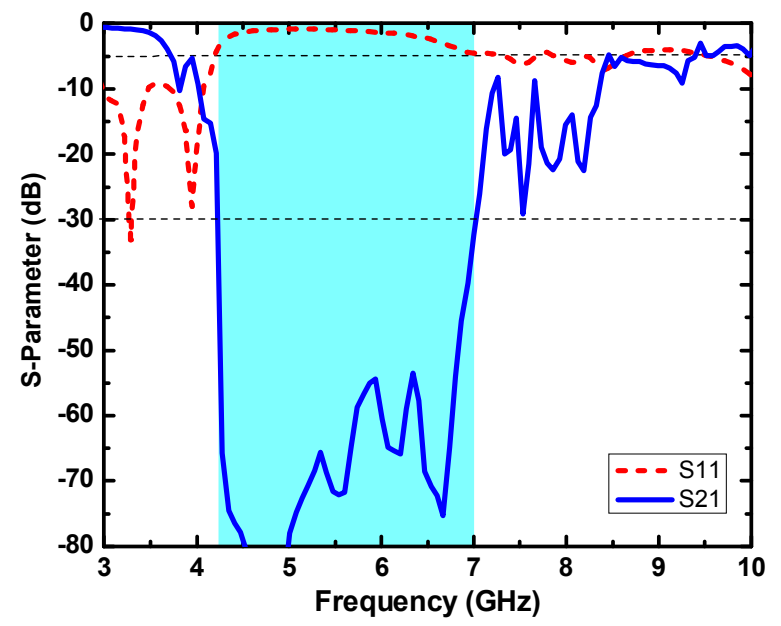

(a)

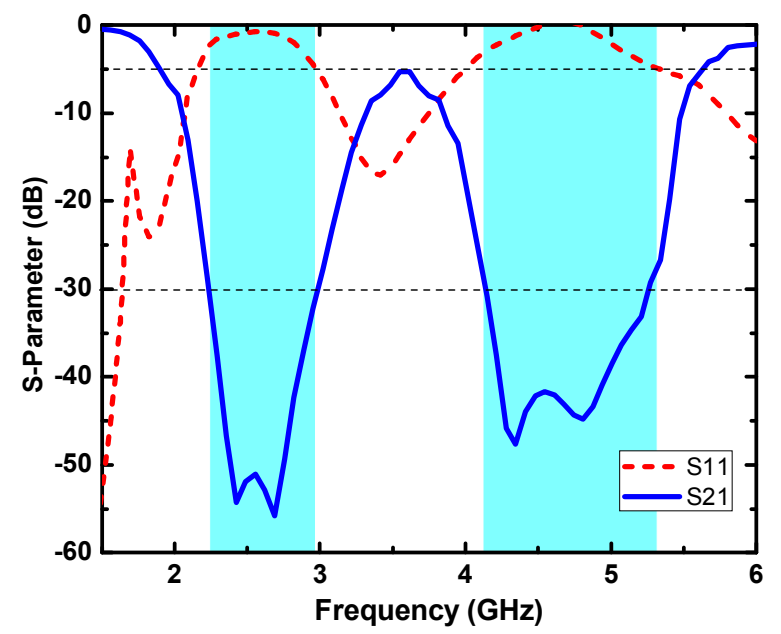

(b)

Figure 5. Operation bandwidth of Hexagonal EBG structure based on Sierpinski triangle at step 2: a) G2 $=0.5 \mathrm{~mm}$ and $\mathrm{b}$ ) $G 2=0 \mathrm{~mm}$

Step 2: As can be seen from Fig. 5a, the EBG structure based on the Sierpinski triangle at step 2 has a lower bandgap than the one in the case of step 1 . The bandwidth of this bandgap covers from 4.22 to 
$6.88 \mathrm{GHz}$. Two bandgaps are defined in Fig. $5 \mathrm{~b}$ when the value of $G 2$ is set at $0 \mathrm{~mm}$. The lower bandgap covers from $2.25 \mathrm{GHz}$ to $2.96 \mathrm{GHz}$ and the higher one spans from $4.14 \mathrm{GHz}$ to $5.34 \mathrm{GHz}$. In this case, both of bandgapsare defined not clear, because the ranges of frequencies of $\mathrm{S} 11$, which are up to $-5 \mathrm{~dB}$, are not flat.

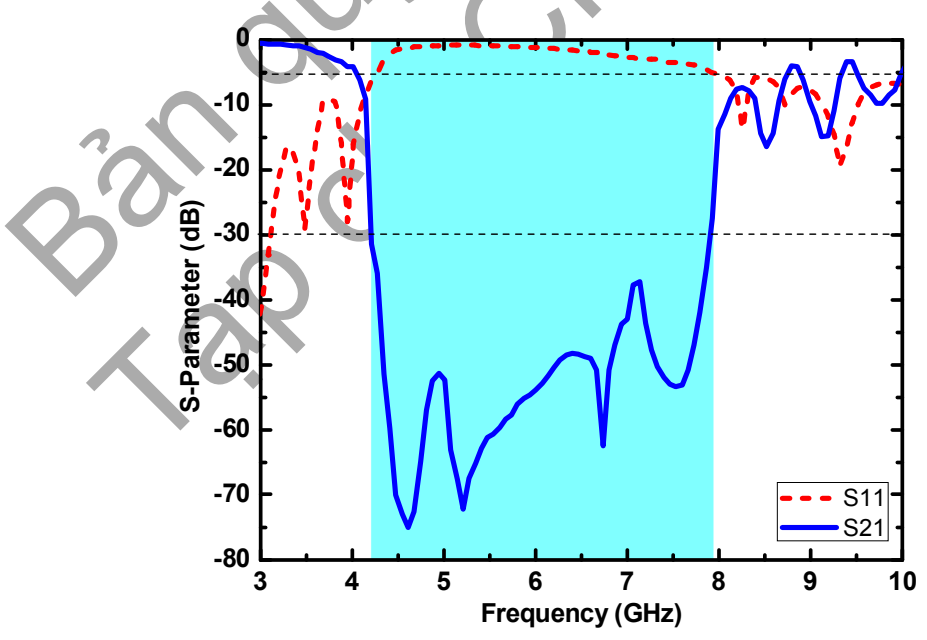

(a)

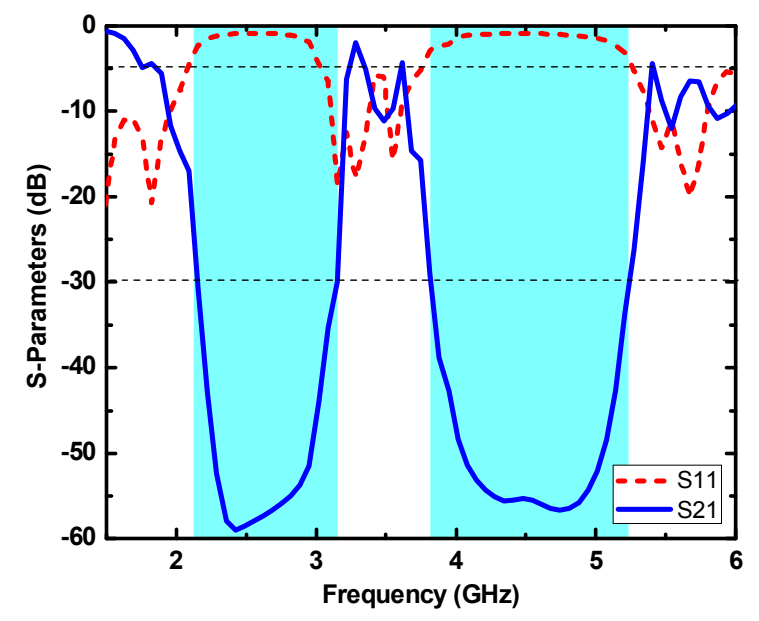

(b)

Figure 6. Operation bandwidth of Hexagonal EBG structure based on Sierpinski triangle at step 3: a) G2 $=0.5 \mathrm{~mm}$ and $b$ ) $G 2=0 \mathrm{~mm}$

Step 3: As can be obverses from Fig. 6a, the bandgap of EBG structure at step 3 is larger than the one in the case of step 2 as $G 2$ is set at $0.5 \mathrm{~mm}$. This bandgap spans the frequencies from 4.32 to $7.92 \mathrm{GHz}$. Moreover, when the $\mathrm{G} 2$ is equal to $0 \mathrm{~mm}$ the bandgaps are determined easier than the one in the case of step 2. From Fig. 6b, the curve of S11 is quite flat in two bandgaps. The first bandgap spans the frequencies from 2.15 to $3.02 \mathrm{GHz}$; the second one has the bandwidth that is covered from $3.81 \mathrm{GHz}$ to 5.20 $\mathrm{GHz}$.

Next, the bandgap properties of the EBG structures at step 4 are considered by investigating the effect of parameters such as the size of unit cell $W$, the gap between two adjacent unit cells $G 1$ to the bandgaps in both cases of DEBG and BEBG.

\section{B. Broadband $E B G(B E B G)$}

General, in order to obtain a wider EBG operation bandwidth, it is necessary to increase $L$ and reduce $C$. Equivalent inductance $L$ can be increased using a thicker dielectric substrate and including in the geometry narrow. Equivalent capacitance $C$ can be reduced by reducing substrate's relative dielectric permittivity and increasing the gap between the metallization edge and the unit-cell edge (and so the gap between adjacent unit cells). Moreover, the thickness of substrate has to be adopted in order to obtain both compact size and broad EBG operation bandwidth.

In the EBG design procedure, if the dielectric material and its thickness have been chosen in this analysis, the total equivalent inductance $L$ cannot be altered. Therefore, only capacitance $C$ can be changed. Regarding the total equivalent $C$ of the unit cell, the contribution due to the gap between adjacent EBG cells (coplanar $C$ ) varies as the distance between unit cells reduces (which increases $C$ ) and also the distance between the metallization equilateral triangle unit inside the EBG unit cells (which decreases coplanar $C$ ). More important is that, in addition, the contribution due to the capacitance between unit-cell metallization and the unit-cell ground plane (parallel plates) is reduced for smaller unit-cell size $(W)$.

Finally, the wider EBG operation bandwidth for smaller unit-cell size $(W)$ of this novel design is due to more significantly decreased $C$ values of the parallel equivalent circuit. Moreover, the EBG operation 
bandwidth is also increased by reducing the relative dielectric permittivity for a give unit-cell size.

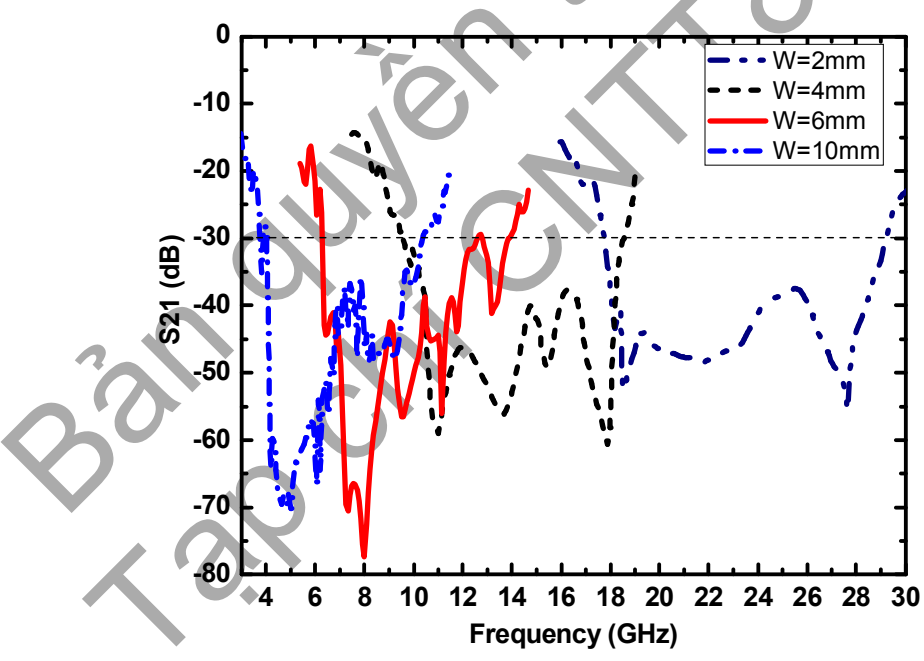

Figure 7. EBG operation bandwidth variations versus EBG unit-cell size $(W)$

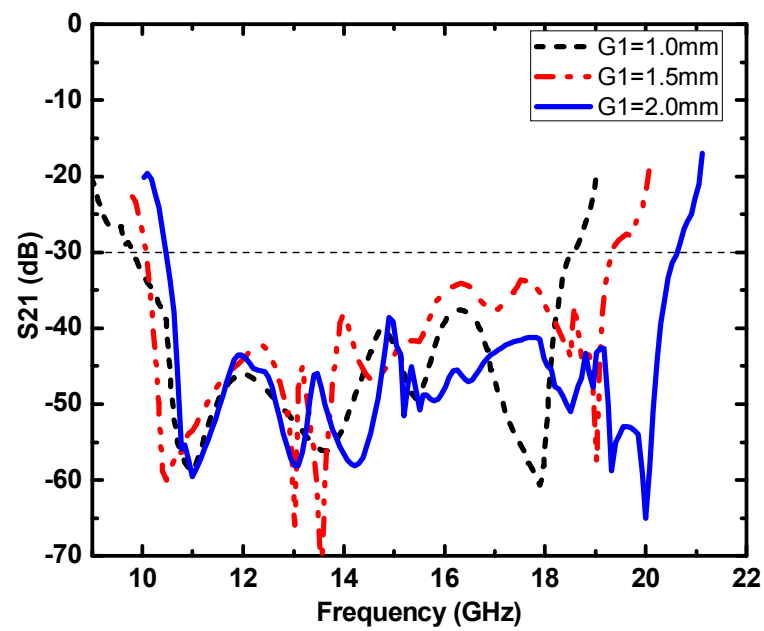

Figure 8. BEBG operation bandwidth variations versus the gap between adjacent EBG cells (G1) while the unit-cell $(W)$ is fixed at $4 \mathrm{~mm}$

In order to analyse the effect of the parameters of dimension to the total equivalent capacitance $C$, the unit-cell $(W)$ and the gap between adjacent EBG units (G1) are investigated while other parameters are constant. The unit cell size $(W)$ is very important parameter to take effect on the bandgap, which affect the equivalent capacitance of the LC resonant circuit of the EBG structure. Firstly, we investigate the bandgap of the EBG structure with different values of
$W$. The unit cell size is increased from $2 \mathrm{~mm}$ to 10 $\mathrm{mm}$ while other parameters of the EBG structure are unchanged. The bandgap moves to lower frequency region with patch size increasing and the bandgap becomes narrow. From Fig. 7, we can see that the equivalent capacitance diminished when the patch size increases, so the resonant frequency is decreased. Since the bandgap width is proportional to $L / C$, the bandgap becomes narrow. The detail of this is shown in Table I.

Table 1. Parametric analysis of ebg unit cell

\begin{tabular}{|c|c|c|c|c|}
\hline $\begin{array}{c}\text { Unit cell } \\
\text { size }(\mathrm{mm})\end{array}$ & $\begin{array}{c}\text { Thickness } \\
(\mathrm{mm})\end{array}$ & $\varepsilon_{\mathrm{r}}$ & $\begin{array}{c}\text { Bandwidth } \\
(\%)\end{array}$ & $\begin{array}{c}\text { Resonance } \\
\text { Frequency } \\
(\mathrm{GHz})\end{array}$ \\
\hline $2(\lambda / 6.4)$ & $1.6(\lambda / 8)$ & 4.4 & 49 & 23.50 \\
\hline $4(\lambda / 5.2)$ & $1.6(\lambda / 13)$ & 4.4 & 65 & 14.05 \\
\hline $6(\lambda / 4.9)$ & $1.6(\lambda / 5)$ & 4.4 & 76 & 10.06 \\
\hline $10(\lambda / 4.0)$ & $1.6(\lambda / 4)$ & 4.4 & 87 & 7.20 \\
\hline
\end{tabular}

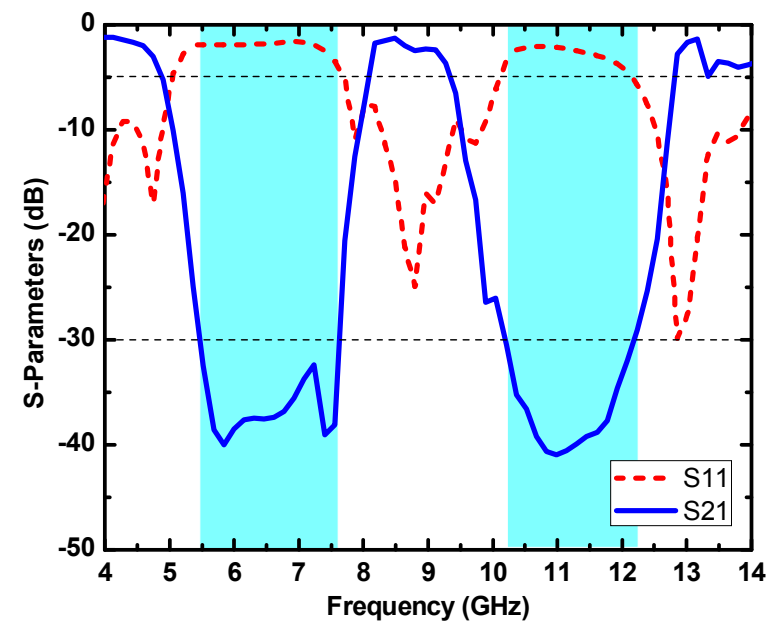

Figure 9. DEBG operation bandwidth with $G 2$ is equal to $0 \mathrm{~mm}$ and $W$ is equal to $4 \mathrm{~mm}$ while the other parameters are fixed

Moreover, the effect of distance between adjacent EBG cells (G1) on the width of the bandgap is also investigated, and the simulated results are shown in Fig. 8. As can be observed from Fig. 10 that the EBG operation bandwidth becomes wider by increasing the gap G1 between adjacent EBG cells and the bandgap move to the higher frequency area. It is shown that the coplanar C is increased with the higher values of G1. 


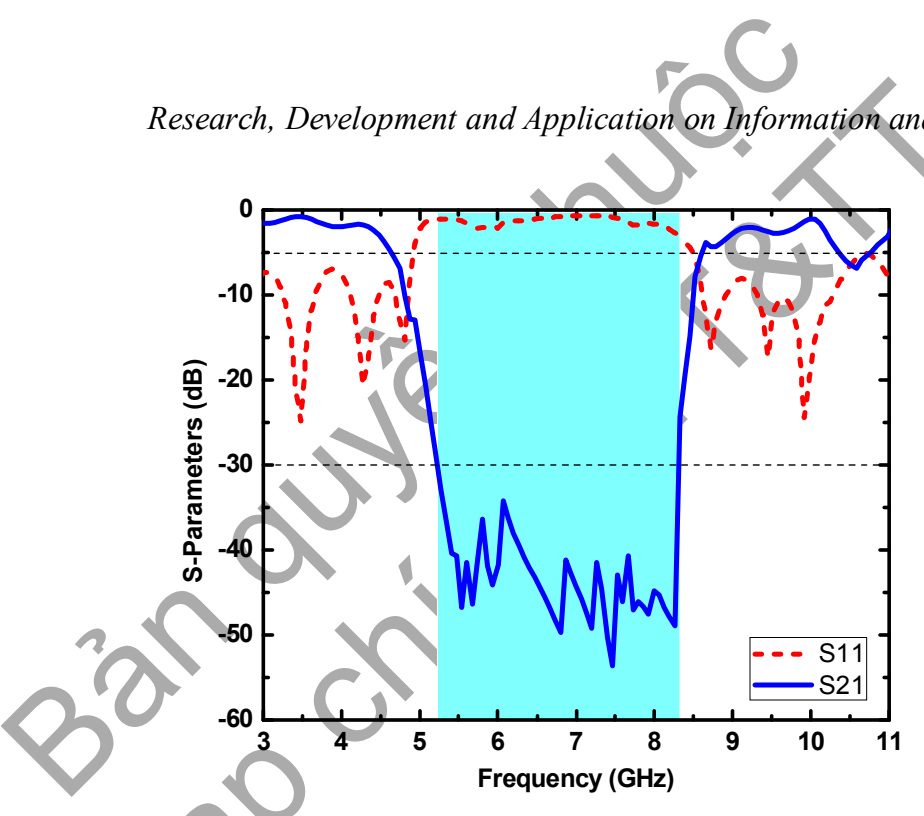

Figure 10. Simulated results of scatter parameters of conventional mushroom-like EBG structure $(W=10 \mathrm{~mm})$

\section{Dual-band EBG (DEBG)}

Table 2. Relative Operation Bandwidth of EBG Structure for various $W$ while $G 2$ is fixed at $0 \mathrm{~mm}$ and $0.5 \mathrm{~mm}$

\begin{tabular}{|c|c|c|c|}
\hline \multirow{2}{*}{$\begin{array}{c}\text { Unit cell } \\
\text { size } W(\mathrm{~mm})\end{array}$} & \multicolumn{3}{|c|}{$G 2$} \\
\cline { 2 - 4 } & $\begin{array}{c}0.5 \mathrm{~mm} \\
\text { Bandgap } \\
(\mathrm{GHz})\end{array}$ & $\begin{array}{c}\text { Bandgap 1 } \\
(\mathrm{GHz})\end{array}$ & $\begin{array}{c}\text { Bandgap 2 } \\
(\mathrm{GHz})\end{array}$ \\
\hline 2 & $17.74-29.25$ & $10.77-14.47$ & $18.80-21.95$ \\
\hline 4 & $9.48-18.62$ & $5.51-7.73$ & $10.20-12.33$ \\
\hline 6 & $6.23-13.88$ & $3.72-5.07$ & $6.72-8.53$ \\
\hline 10 & $4.05-10.35$ & $2.06-3.08$ & $3.71-5.26$ \\
\hline
\end{tabular}

In this section, the gap $G 2$ between two adjacent Sierpinski triangles inside the EBG unit cell is also investigated while other parameters are constant. From Fig.9, it can be seen that two bandgaps are determined by transmission coefficient. The unique characteristic of this design that the structure can be transformed the bandwidth from broadband into dualband when $G 2$ is equal to zero. When the unit cell size $W$ is set at $4 \mathrm{~mm}$, the first bandgap spans from $5.51 \mathrm{GHz}$ to $7.73 \mathrm{GHz}$. The bandwidth of the second one is spreading from $10.20 \mathrm{GHz}$ to $12.18 \mathrm{GHz}$. This can be indicated more clearly in Table II. It can be observed from Table II that these bandgaps move forward to the lower frequency regions. It is clear that when the value of $G 2$ greater than zero, two above bandgaps are moving to overlap each other and the broader bandgap can be obtained.

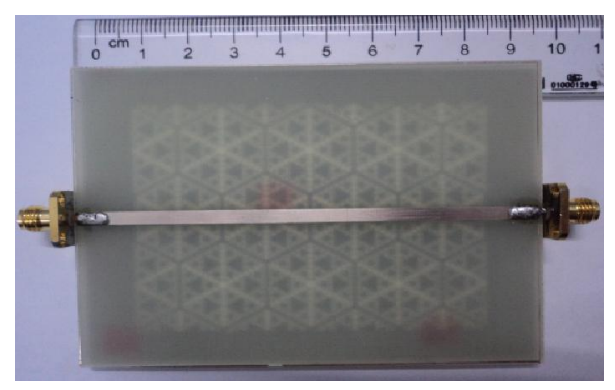

(a)

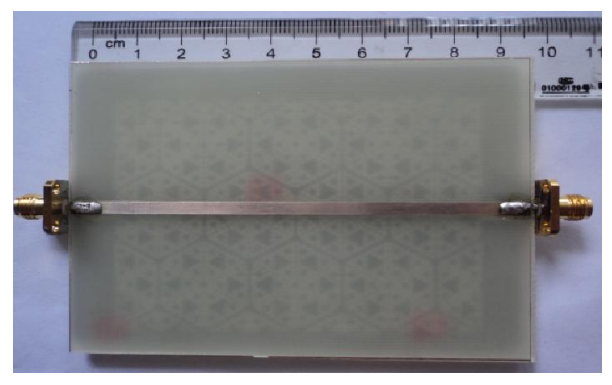

(b)

Figure 11. Photos of the proposed structures with suspended microstrip: (a) array of $3 \times 4$ DEBG cells and (b) array of $3 \times 4$ BEBG cells

\section{Conventional Mushroom-like EBG}

In order to verify the bandgap characteristic of the broadband EBG structure (BEBG), an array of $3 \times 4$ unit cells of hexagonal mushroom-like EBG is investigated (see Fig. 3a). This EBG is also constructed on a dielectric substrate with a relative permittivity of 4.4 and a thickness of $1.6 \mathrm{~mm}$. The unit cell dimension is fixed at $10 \mathrm{~mm}$. The simulated result of transmission coefficient is shown in Fig. 10. The bandgap is centered at $6.77 \mathrm{GHz}$ and the bandgap bandwidth is from $5.22 \mathrm{GHz}$ to $8.32 \mathrm{GHz}$, achieve the bandgap bandwidth about $46 \%$. As can be observed from Table 1, the bandgap bandwidth of the BEBG structure at $\mathrm{W}=10 \mathrm{~mm}$ is much larger $(87 \%)$ than that of the conventional EBG at the same dimension of the unit cell. 


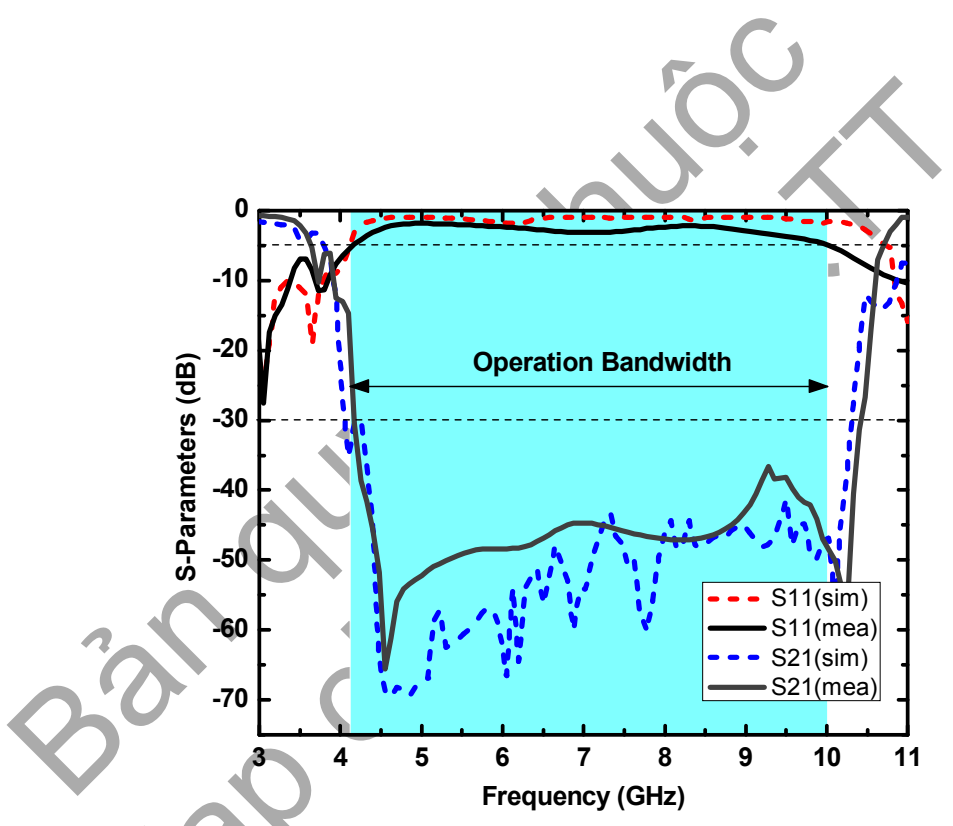

(a)

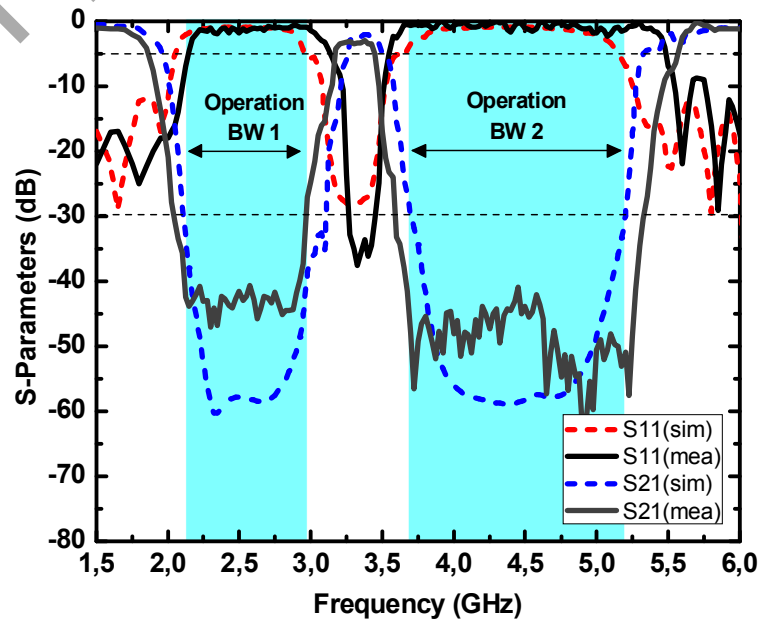

(b)

Figure 12. EBG operation bandwidth $(W=10 \mathrm{~mm})$ : (a) $B E B G$ and (b) $D E B G$

\section{EXPERIMENTAL RESULTS}

Both schematics of proposed EBG structures were simulated and take into comparison with the measurement of the fabrication. The microstrip lines are soldered with SMA connector to measure the scatter parameters. The experimentally measured of scatter parameters of the arrays are performed by Anritsu 37369D network analyzer. Photos of the proposed EBG structures are shown in Fig. 11. The simulated and the measured results of the parameters of BEBG structure and DEBG structure are shown in Fig. 12a and Fig. 12b, respectively.

It is very efficient to determine the bandgap by the transmission curves. From the measurement of S11 with the criteria of $-5 \mathrm{~dB}$, the operation bandwidth can be determined easily when the transmission coefficient S21 is below $-30 \mathrm{~dB}$. As can be shown from Fig. 12a, the BEBG introduce a bandgap between the frequencies $4.15 \mathrm{GHz}-10 \mathrm{GHz}$ with the criteria of $-30 \mathrm{~dB}$, from that the electromagnetic wave cannot propagate. The bandgap bandwidth is about $83 \%$. The reason is that the surface impedance of the EBG structure becomes very high within the bandgap. From Fig. 12b, two distinct bandgaps of DEBG structure are defined in which the first bandgap ranging from 2.17 to $2.97 \mathrm{GHz}$ and the bandwidth of the second bandgap is spreading from 3.58 to 5.32 GHz. The bandgap bandwidths of the DEBG structure are $32 \%$ and $39 \%$, respectively. It can be observed from Table III that the measured results show good agreement with the simulated results.

Table 3. Operation Bandwidth of Proposed EBG Structures with $\mathrm{W}=10 \mathrm{~mm}$

\begin{tabular}{|c|c|c|c|}
\hline \multirow{3}{*}{ Methods } & \multicolumn{3}{|c|}{$G 2$} \\
\hline & \multirow{2}{*}{$\begin{array}{c}0.5 \mathrm{~mm} \\
\text { Bandgap } \\
(\mathrm{GHz})\end{array}$} & \multicolumn{2}{|c|}{$0 \mathrm{~mm}$} \\
\hline & & $\begin{array}{c}\text { Bandgap } 1 \\
(\mathrm{GHz})\end{array}$ & $\begin{array}{c}\text { Bandgap } 2 \\
(\mathrm{GHz})\end{array}$ \\
\hline Simulated & $\begin{array}{c}4.05-10.35 \\
(87)\end{array}$ & $\begin{array}{c}2.06-3.08 \\
(40)\end{array}$ & $\begin{array}{c}3.71-5.26 \\
(35)\end{array}$ \\
\hline Measured & $\begin{array}{c}4.15-10.00 \\
(83)\end{array}$ & $\begin{array}{c}2.17-2.97 \\
(32)\end{array}$ & $\begin{array}{c}3.58-5.32 \\
\text { (39) }\end{array}$ \\
\hline
\end{tabular}

\section{APPLICATION TO PATCH ANTENNAS}

In this section, two proposed EBG structures will be integrated to a patch antenna for enhancing the radiation properties of the antenna. A patch antenna is designed as the reference antenna, which is on a classical ground plane, as shown in Fig.13a. The patch antenna is printed on a dielectric substrate with a relative permittivity of 4.4 , thickness of $1.6 \mathrm{~mm}$ and a size ofWs $\times$ Ws $\mathrm{mm}^{2}$. While, Fig. 13b and Fig. 13c shows two other antennas which are designed with a BEBG and DEBG replacing the conducting ground plane. For comparison purposes, all parameters of the proposed antennas are consistent. The resonant 
frequency of the antenna at $5 \mathrm{GHz}$ is set within the surface wave bandgap of the Fractal-EBGs. In order to effectively suppress the surface waves, three rows of EBG cells are used in the design

Figure 14 shows the simulated S11 of three antennas. The simulated results indicate that the S11 is $-21.85 \mathrm{~dB}$ for the reference antenna, $-29.82 \mathrm{~dB}$ for the BEBG antenna and $-27.94 \mathrm{~dB}$ for the DEBG antenna. It indicates that the reference antenna is about $7.97 \mathrm{~dB}$ and $6.09 \mathrm{~dB}$ greater than the BEBG and DEBG antenna, respectively. Figure 14 illuminates that the EBG structures are used to the antenna ground plane, reducing the antenna input impedance loss and improving the antenna radiation power.

Figure 15 shows the simulated radiation patterns of three antennas in both planes $\mathrm{XZ}$ and $\mathrm{YZ}$ at $5 \mathrm{GHz}$. The reference antenna shows large radiationin the backward direction, and the antenna atthe FractalEBG ground plane produces a lower back lobe, with less power wastedin the backward direction. As shown in Figure 15, in the radiation pattern of the patch antenna with DEBG and BEBG, the back lobe is reduced by $9.16 \mathrm{~dB}$ and $8.03 \mathrm{~dB}$, respectively. The radiation pattern of the antenna is improved effectively.

\section{Conclusion}

A flexible EBGs design is presented using Sierpinski Gasket triangles, which are arrangedto repeat each $60^{\circ}$ to form the hexagonal EBG unit cell. Two EBG structures, which have broadband and dual bandgap, are proposed by setting the value of the gap between two adjacent Sierpinski inside the unit cell is equal to $0 \mathrm{~mm}$ and greater than $0 \mathrm{~mm}$. The suspending microstrip method is used to simulate the scatter coefficients of the EBG structure. The bandwidth of BEBG structure is much larger than that of the conventional EBG. The results show a good agreement between simulations and measurements. Due to several advantages of the structures such as using inexpensive dielectric substrate FR4 and planar structure, the EBG structures are used as a high impedance ground plane for a patch antenna. As the results, the antenna with a Fractal-EBG ground plane has already reduced significant the back lobe and improved the radiation efficiency in comparison to the one with the conducting ground plane.

\section{REFERENCES}

[1] F. R. Yang, K. P. Ma, Y. Qian, and T. Itoh, "A uniplanar compact photonic-bandgap (UC-PBG) structure and its applications for microwave circuits," IEEE Trans. Microwave Theory \& Tech., Vol. 47, No. 8, pp. 1509-1514, Aug. 1999.

[2] B.Q. Lin, Q.R. Zheng and N.C. Yuan, "A novel planar PBG structure for size reduction," IEEE Microw. Wireless Compon. Lett., vol. 16, no. 5, pp. 269-271, May. 2006

[3] L. Yang, M. Fan, F. Chen, J. She, and Z. Feng, "A Novel Compact Electromagnetic-Bandgap (EBG) Structure and Its Applications for Microwave Circuits," IEEE Trans. Microw. Theory Tech., vol.53, no. 1, pp. 183-190, Jan. 2005.

[4] L. J. Zhang, C.-H. Liang, L. Liang, and L. Chen, "A Novel Design Approach For Dual-Band Electromagnetic Bandgap Structure" Progress In Electromangetics Research M, vol. 4, 81-91, 2008.

[5] J.D. Joannopoulos, R. D. Meade, and J.N. Winn, "Photonic Crystals," Princeton, NJ: Princeton Univ. Press, 1995.

[6] E.Yablonovitch, "Photonic bandgap structure," , J. Opt. Soc. Amer. B, Opt. Phys., Vol.10,pp.283295,Feb.1993.

[7] D. Sievenpiper, L. Zhang, R. Broas, N. Alexopolous, and E. Yablonovitch, "High-impedance frequency selective surface with forbidden frequency band," IEEE Trans. Microwave Theory Tech. ,Vol. 47, pp.2059- 074, Nov.1999.

[8] A. Stark, S. Prorok, and A. F. Jacob, " Broadband EBG structures with compact unit cell,"Proceedings of the $38^{\text {th }}$ European Microwave Conference, October 2008, Amsterdam, The Netherlands.

[9] L. Liang, C.H. Liang, X.W.Zhao, and Z.J.Su, "A novel broadband EBG using multi-period mushroom-like structure,"Proceedings of International Conference on Microwave and Milimetter Wave Technology, April 2008, Nanjing, China.

[10] R.Coccioli, F. R. Yang, K. P. Ma and T. Itoh, "Aperture-coupled patch antenna on UC-PBG substrate," IEEE Trans. Microwave Theory Tech. ,vol.47, pp.2123-2130,Nov.1999.

[11] M. Rahman and M. A. Stuchly, "Circularly Polarised Patch Antenna With Periodic Structure," in IEEE Proc. Microwave Antennas \& Propagation, vol. 149, Jun 2002, pp. 141-146.

[12] M. F. Abedin, M. Z. Azad, and M. Ali, "Wideband Smaller Unit-Cell Planar EBG Structure and Their Application" IEEE Trans. Antennas Propagat., vol. 56, no. 3, pp. 903-908, Mar. 2008. 
[13] W. J. Krzysztofik, "Modified Sierpinski Fractal Monopole for ISM-Bands Handset Applications," IEEE Trans. Antennas Propagat., vol. 57, no. 3, pp. 606-615, Mar.2009.

[14] Fan, R. H. M. Y., Z. H. Feng, and X. X. Zhang, "New method for 2D-EBG structure research," J. Infrared Millimeter Waves, Vol. 22, No. 2, 2003.

[15] T. Masri, M.K.A. Rahim, and M.N.A. Karim, "A Novel 2D Sierpinski Gasket Electromagnetic Bandgap Structure for Multiband Microstrip Antenna," Proceeding of Asia-Pacific Conference on Applied Electromagnetics, December 2007, Melaka, Malaysia.

[16] R. Kumar, G. Mathai, and J.P. Shinde, "Design of Compact Multiband EBG and Effect on Antenna Performance," International Journal of Recent Trends in Engineering, Vol. 2, No. 5, pp. 254-258, Nov. 2009.

\section{AUTHOR'S BIOGRAPHIES}

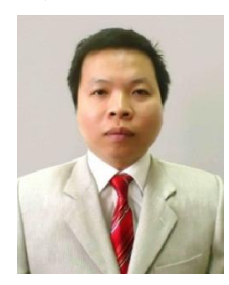

Huynh Nguyen Bao Phuong received the B.E and M.Sc. degrees in 2003 and 2007, respectively, from School of Electronics and Telecommunications, Hanoi University of Science and Technology, Vietnam. Now he is a Lecturer of the Faculty of Technique and Technology, Quynhon University, Vietnam. Currently, he is working toward Ph.D degree at the School of Electronics and Communications, Hanoi University of Science and Technology, Vietnam.

His research interests are high impedance surfaces (HIS), ultra wideband (UWB), microstrip and metamaterial antennas.

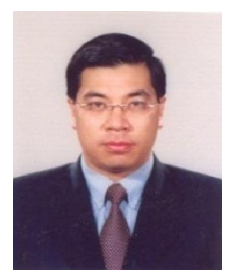

Tran Minh Tuan (Assoc. Prof. Ph.D) was born in Hanoi - Vietnam in 1970. He received the B.E degree and M.E degree in Satellite Communications from Moscow Institute of Technology in Russia in 1994 and in 1995, respectively. In 2004, he received Ph.D degree in antenna and radiowave propagation in Hanoi University of Science and Technology, Vietnam. Now he is Vice President of National Institute of Information and Communications Strategy, Ministry of Information and Communications of Vietnam.

His current research interests includes: master plans and strategies of telecommunications and IT development in Vietnam, radiowave broadcasting and propagation, slowwave structures, slotted, leaky-wave and microstrip antennas, CAD software for radio-wave structures, slotted, leaky-wave and microstrip antennas etc.

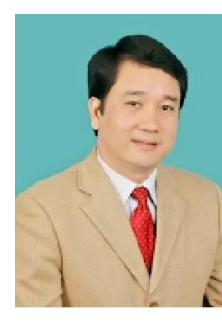

Dao Ngoc Chien received the Diploma of Engineer in 1997 from the Department of Telecommunication Systems, School of Electronics and Telecommunications, Hanoi University of Science and Technology, where in the same year he became a Teaching Assistant. He received M.Sc. and Ph.D. degrees in 2002 and 2005, respectively, from the Department of Electronics and Computer Engineering, Gifu University, Japan. At the Department of Telecommunication Systems, School of Electronics and Telecommunications, Hanoi University of Science and Technology, he worked as the Senior Lecturer from 2005 to 2011, and is currently the Contracted Lecturer. He has been appointed to Associate Professor since November, 2010.

His research interests include computational electromagnetics based on MoM and FDTD methods, analysis and design of modern antennas and of nanometric integrated optical circuits based on the surface plasmon polaritons. He has been a reviewer for several journals/transactions of Optical Society of America (OSA), Institute of Electrical and Electronics Engineers (IEEE), Elsevier, and American Geophysical Union (AGU), as well as for a number of conferences. He has been a member of IEEE, OSA and REV. 